OPEN ACCESS

Edited by:

Luigi Iuliano,

Sapienza University of Rome, Italy

Reviewed by:

Nada A. Abumrad,

Washington University in St. Louis,

United States

Andrey Turchinovich,

German Cancer Research Center

(DKFZ), Germany

${ }^{*}$ Correspondence:

Siti Aishah Sulaiman

sitiaishahsulaiman@ukm.edu.my

Specialty section:

This article was submitted to

Lipid and Fatty Acid Research,

a section of the journal

Frontiers in Physiology

Received: 03 December 2018

Accepted: 04 March 2019

Published: 19 March 2019

Citation:

Sulaiman SA, Muhsin NIA and Jamal $R$ (2019) Regulatory

Non-coding RNAs Network

in Non-alcoholic Fatty Liver Disease.

Front. Physiol. 10:279.

doi: 10.3389/fphys.2019.00279

\section{Regulatory Non-coding RNAs Network in Non-alcoholic Fatty Liver Disease}

\author{
Siti Aishah Sulaiman*, Nor I. A. Muhsin and Rahman Jamal \\ UKM Medical Molecular Biology Institute, Universiti Kebangsaan Malaysia, Kuala Lumpur, Malaysia
}

Non-alcoholic fatty liver disease (NAFLD) spectrum comprises simple steatosis and nonalcoholic steatohepatitis (NASH) that can lead to fibrosis and cirrhosis. The patients usually have no history of excessive alcohol consumption and other etiologies that can cause fatty liver. Understanding of the pathophysiology of NAFLD has revealed that noncoding RNAs (ncRNAs) play significant roles in modulating the disease susceptibility, pathogenesis and progression. Currently, the ncRNAs are grouped according to their sizes and their regulatory or housekeeping functions. Each of these ncRNAs has a wide range of involvement in the regulation of the genes and biological pathways. Here, we briefly review the current literature the regulatory ncRNAs in NAFLD pathogenesis and progression, mainly the microRNAs, long non-coding RNAs and circular RNAs. We also discuss the co-regulatory functions and interactions between these ncRNAs in modulating the disease pathogenesis. Elucidation of ncRNAs in NAFLD may facilitate the identification of early diagnostic biomarkers and development of therapeutic strategies for NAFLD.

Keywords: IncRNAs, circRNAs, microRNAs, NAFLD, NASH, biomarker

\section{INTRODUCTION}

Non-alcoholic fatty liver disease (NAFLD) is one of the common chronic liver diseases. It is characterized by the presence of hepatic steatosis ( $>5-10 \%$ of fatty hepatocytes) in an individual without a history of excessive alcohol consumption and other disease etiologies that can cause fatty liver (Araújo et al., 2018). Currently, NAFLD prevalence in the general population is about $24-25 \%$ (Bedogni et al., 2005; Younossi et al., 2016, 2017), with the highest prevalence reported in South America and the Middle East, followed by Asia, United States, and Europe (Younossi et al., 2016). In parallel with the increasing incidence of obesity and diabetes, NAFLD will be the leading cause of cirrhosis and HCC in the next 5 years, surpassing hepatitis infection (Streba et al., 2015). Therefore, research on NAFLD pathogenesis, early diagnosis, and biomarkers as well as identification of the therapeutic targets for NAFLD are necessary to reduce the burden of this disease.

Non-alcoholic fatty liver disease is a spectrum disease, in which untreated patients with liver steatosis or non-alcoholic fatty liver (NAFL) can progress to non-alcoholic steatohepatitis (NASH) that further increases the risk of developing cirrhosis and hepatocellular carcinoma (HCC) (Streba et al., 2015). Similar to other metabolic diseases, NAFLD is a multi-factorial disease in which the genetic predisposition, environmental exposures and lifestyle factors can modulate susceptibility to the disease and progression (Wruck et al., 2017). Previous studies have shown that non-coding RNAs (ncRNAs) are implicated in the etiology of NAFLD and possibly be the key mediators 
in its pathogenesis (Jiang and Zhang, 2017; Lin et al., 2017; Hanson et al., 2018; Turchinovich et al., 2018). These ncRNAs are constitutively expressed and can regulate biological processes, genes and proteins (Yamamura et al., 2018). In this minireview, we aimed to discuss the role of ncRNAs in NAFLD development and progression, as well as the co-regulatory interaction between these ncRNAs.

\section{PATHOGENESIS: FATTY LIVER PROGRESSION TO NASH AND FIBROSIS}

Fatty liver is defined by the accumulation of the triglycerides (TGs) in hepatocytes, due to an imbalance between energy consumption [free fatty acids (FFAs) uptake and de novo lipogenesis] and energy metabolism (fatty acid oxidation, lipoprotein packaging and transport) (Peverill et al., 2014; Manne et al., 2018). For most of the patients, this fatty liver condition is non-pathogenic and can be reversed by appropriate interventions, though about $20-30 \%$ of them will progress to NASH (Younossi et al., 2017). The exact mechanisms of how fatty liver progresses to NASH is not fully understood. Oxidative stress and inflammation induced by lipotoxicity appear to be the key mechanism in NASH progression (Peverill et al., 2014; Manne et al., 2018). A "two-hit" hypothesis proposes that NASH progression starts with the first hit of insulin resistance that contributes to hepatic steatosis (Gentile and Pagliassotti, 2008) and the second hit of the inflammatory cytokines induced by the oxidative stress (Sumida et al., 2013). However, recent findings have shown that NASH progression is far more complex and the "two-hit" hypothesis is not sufficient to describe the pathogenesis. The interactions and cross-talks between the liver parenchymal (hepatocytes) and non-parenchymal cells [hepatic stellate cells (HSCs)], Kupffer cells (stellate macrophages), and various immune cells are recently recognized to participate in NASH progression (Magee et al., 2016). Excessive TGs accumulation promotes the hepatocellular injury (ballooning) which will stimulate the inflammatory response and activates the liver immune cells and HSCs (Magee et al., 2016). In response to hepatocyte injury, HSCs transform or activate into a myofibroblast phenotype and promotes the secretion of cytokines and components of the extracellular matrix (ECM) to protect the liver (Magee et al., 2016). Therefore, in the setting of chronic inflammation, prolonged secretion of ECM components results in hepatic scarring and fibrosis (Friedman, 2008). Due to these complicated interactions, a "multiple-hit" hypothesis is more acceptable to describe NASH progression (Tilg and Moschen, 2010). In this "multiple-hit" model, several dysregulated pathways and insults can act in parallel in an individual in combination with genetic predisposition (Tilg and Moschen, 2010), thus increases the risk of developing NASH. Due to the word limit imposed, this review will not discuss NAFLD/NASH pathogenesis in details, as previous reviews have covered the topic extensively, including the various mechanisms, pathways and genetic factors involved (Tilg and Moschen, 2010; Sumida et al., 2013; Peverill et al., 2014; Magee et al., 2016; Ananthanarayanan, 2018; Eslam et al., 2018; Ibrahim et al., 2018;
Manne et al., 2018). Despite the fact that NASH patients have a greater risk of developing end-stage liver diseases (Streba et al., 2015), it is unclear why some NAFL patients progress to NASH while some others do not.

\section{NON-CODING RNAs IN NAFLD}

Non-coding RNAs (ncRNAs) refers to a group of RNAs that do not encode for a protein, and most of these ncRNAs are the products of alternative splicing with the larger transcripts become the precursors for smaller ncRNAs (Djebali et al., 2012). Initially, these ncRNAs are considered to be the genome "junks," but in recent years, ncRNAs are shown to be involved in various cellular processes and disease stages, with emerging evidence of their interactions with each other to form a complex regulatory network (Yamamura et al., 2018). In general, ncRNAs belong into two groups according to their lengths, i.e., the small ncRNAs ( $<200$ nucleotides) and the long ncRNAs ( $>200$ nucleotides) (Djebali et al., 2012). Within these two groups, they are further characterized according to their functions. Among the small ncRNAs, the transfer RNAs, small nucleolar RNAs and small nuclear RNAs are known as the small housekeeping ncRNAs, whereas PIWI-interacting RNAs (piRNAs), circular (circRNAs) and microRNAs (miRNAs) are known as the small regulatory ncRNAs (Djebali et al., 2012). Similarly, for the long ncRNAs, the ribosomal RNA is the housekeeping long ncRNA, whereas the long ncRNAs (lncRNAs) and enhancer RNAs (eRNAs) are known as the long regulatory ncRNAs (Djebali et al., 2012; Hon et al., 2017).

\section{MicroRNAs (miRNAs)}

MicroRNAs (miRNAs) are highly conserved short singlestranded ncRNAs $(\sim 18-22$ nucleotides) that can regulate gene expression via specific complementary binding to target mRNA, and results in either mRNA degradation (perfect binding) or translational suppression (imperfect binding), though this silencing of mRNA expression can be reversed (Vishnoi and Rani, 2017). MiRNAs are the most studied ncRNAs with their biogenesis and processing are well-defined (O'Brien et al., 2018). Majority of the miRNAs are produced via the canonical pathway that starts with miRNA transcription step thus producing long primary transcripts (pri-miRNAs) (O'Brien et al., 2018). The primiRNAs are then processed by RNase III-type enzyme, Drosha to generate the hairpin precursors (pre-miRNAs). These premiRNAs are then exported to the cytoplasm, where they are further processed into mature miRNA duplex by the RNase III protein Dicer (O'Brien et al., 2018). Finally, one of the miRNA duplex strands is incorporated into the RNA-induced silencing complex (RISC), to exert their regulatory function (O'Brien et al., 2018). A previous review has extensively discussed miRNA biogenesis and their regulatory mechanism in details, including the non-canonical biogenesis pathway and miRNA modifications (O'Brien et al., 2018), therefore these topics will not be discussed here. As for NAFLD/NASH, miRNAs roles have been reviewed in terms of the disease pathogenesis, as a biomarker and as a potential therapeutic target (Baffy, 2015; 
Gerhard and DiStefano, 2015; DiStefano and Gerhard, 2016; He et al., 2016; Otsuka et al., 2016; Lin et al., 2017). Hence, this review will only highlight those findings. All the miRNAs identified in circulating samples of the NAFLD/NASH patients are summarized in Table 1.

\section{MicroRNAs in Hepatic Lipid Regulation, Steatosis and NASH}

The most well-known hepatic miRNAs is miR-122 that is highly expressed in the liver, which acts as a beacon for hepatocyte status (Cheung et al., 2008; Hsu et al., 2012; Csak et al., 2015; Pirola et al., 2015) and to preserve adult liver signature profile (Krützfeldt et al., 2005) (Figure 1). Transient inhibition of miR122 expression resulted in a lower cholesterol level, due to increased hepatic fatty acid oxidation (Krützfeldt et al., 2005; Esau et al., 2006; Elmén et al., 2008), and improvement in hepatic steatosis (Esau et al., 2006). However, total knockout of miR-122 expression was detrimental, as it caused higher TGs accumulation and hepatic micro-steatosis that progressed to NASH and fibrosis (Hsu et al., 2012). Consistent with these findings, reduced expression of miR-122 was observed in hepatic tissues of NAFLD and NASH, both in animal models (Alisi et al., 2010; Tryndyak et al., 2012) and human patients (Cheung et al., 2008; Pirola et al., 2015; Aitana et al., 2016; Latorre et al., 2017).

Besides miR-122, several other miRNAs are involved in NAFLD pathogenesis, particularly in liver steatosis (Table $\mathbf{1}$ and Figure 1). One is miR-34a, which was shown to be upregulated in both tissue and serum samples of NAFLD/NASH patients (Cheung et al., 2008; Yamada et al., 2013; Salvoza et al., 2016). miR-34a was shown to downregulate expression of several key genes in NAFLD pathogenesis, including $H N F 4 \alpha$ (Xu Y. et al., 2015), PPAR $\alpha$ and SIRT1 expressions (Ding et al., 2015; Shan et al., 2015). Dysregulation of these genes has been associated with higher TGs accumulation and liver steatosis (Ding et al., 2015; Shan et al., 2015; Xu Y. et al., 2015). Furthermore, this reduction of SIRT1 expression also initiated the activation of pro-apoptotic genes, including P66SHC (Shan et al., 2015) and P53 (Chang et al., 2007; Castro et al., 2013), which could increase hepatocyte susceptibility toward oxidative stress and apoptosis. Another miRNA involved in

TABLE 1 | Reported expression of microRNAs identified in NAFLD, NASH and fibrosis patients.

\begin{tabular}{|c|c|c|c|}
\hline Non-coding RNAs & Liver Disease & Tissue Expression & Circulating Expression \\
\hline \multirow[t]{3}{*}{$\operatorname{miR}-122$} & NAFLD & Reduced (Latorre et al., 2017) & $\begin{array}{l}\text { Increased (Cermelli et al., 2011; Yamada et al., } \\
\text { 2013; Tan et al., 2014; Pirola et al., 2015; Liu et al., } \\
\text { 2016; Salvoza et al., 2016; Brandt et al., 2018) }\end{array}$ \\
\hline & $\mathrm{NASH}$ & $\begin{array}{l}\text { Reduced (Cheung et al., 2008; } \\
\text { Pirola et al., 2015; Aitana et al., } \\
\text { 2016) }\end{array}$ & $\begin{array}{l}\text { Increased (Becker et al., 2015; Pirola et al., 2015; } \\
\text { Liu et al., 2016) }\end{array}$ \\
\hline & Fibrosis & Reduced (Miyaaki et al., 2014) & Reduced (Miyaaki et al., 2014; Akuta et al., 2016) \\
\hline \multirow[t]{2}{*}{ miR-34a } & NAFLD & NA & $\begin{array}{l}\text { Increased (Cermelli et al., 2011; Yamada et al., } \\
\text { 2013; Salvoza et al., 2016) }\end{array}$ \\
\hline & $\mathrm{NASH}$ & $\begin{array}{l}\text { Increased (Cheung et al., 2008; } \\
\text { Aitana et al., 2016) }\end{array}$ & Increased (Liu et al., 2016) \\
\hline $\mathrm{miR}-33 \mathrm{a} / \mathrm{b}$ & $\mathrm{NASH}$ & Increased (Joel et al., 2016) & NA \\
\hline miR-103/miR-107 & NAFLD & NA & Increased (Xu Q. et al., 2015) \\
\hline \multirow[t]{2}{*}{ miR-21 } & NAFLD & NA & Increased (Yamada et al., 2013; Liu et al., 2016) \\
\hline & $\mathrm{NASH}$ & NA & Increased (Becker et al., 2015) \\
\hline \multirow[t]{2}{*}{ miR-192 } & NAFLD & NA & $\begin{array}{l}\text { Increased (Tan et al., 2014; Pirola et al., 2015; Liu } \\
\text { et al., 2016) }\end{array}$ \\
\hline & $\mathrm{NASH}$ & Reduced (Pirola et al., 2015) & $\begin{array}{l}\text { Increased (Becker et al., 2015; Pirola et al., 2015; } \\
\text { Liu et al., 2016) }\end{array}$ \\
\hline \multirow[t]{2}{*}{$\operatorname{miR}-223$} & NAFLD & Reduced & Increased \\
\hline & $\mathrm{NASH}$ & Reduced & Increased (Becker et al., 2015) \\
\hline miR-29c & $\mathrm{NASH}$ & Reduced (Aitana et al., 2016) & NA \\
\hline miR-181d, miR-99a, miR-197 & $\mathrm{NASH}$ & NA & Reduced (Celikbilek et al., 2014) \\
\hline $\operatorname{miR}-16$ & $\mathrm{NASH}$ & NA & Increased (Cermelli et al., 2011; Liu et al., 2016) \\
\hline \multirow[t]{2}{*}{ miR-146b } & NAFLD & Reduced (Latorre et al., 2017) & Increased (Liu et al., 2016) \\
\hline & $\mathrm{NASH}$ & Increased (Cheung et al., 2008) & Reduced (Celikbilek et al., 2014) \\
\hline \multirow{2}{*}{$\begin{array}{l}\text { miR-19a, miR-19b, miR-125, } \\
\text { miR-375 }\end{array}$} & NAFLD & Reduced & Increased (Pirola et al., 2015) \\
\hline & $\mathrm{NASH}$ & Reduced & Increased (Pirola et al., 2015) \\
\hline miR-1290, miR-27b, miR-148a & NAFLD & NA & Increased (Tan et al., 2014) \\
\hline \multirow[t]{2}{*}{ miR-99a } & NAFLD & NA & Increased (Tan et al., 2014) \\
\hline & $\mathrm{NASH}$ & NA & Reduced (Celikbilek et al., 2014) \\
\hline miR-139, miR-30b, miR-442 & NAFLD & Reduced (Latorre et al., 2017) & NA \\
\hline $\operatorname{miR}-451$ & NAFLD & NA & Increased (Yamada et al., 2013) \\
\hline
\end{tabular}




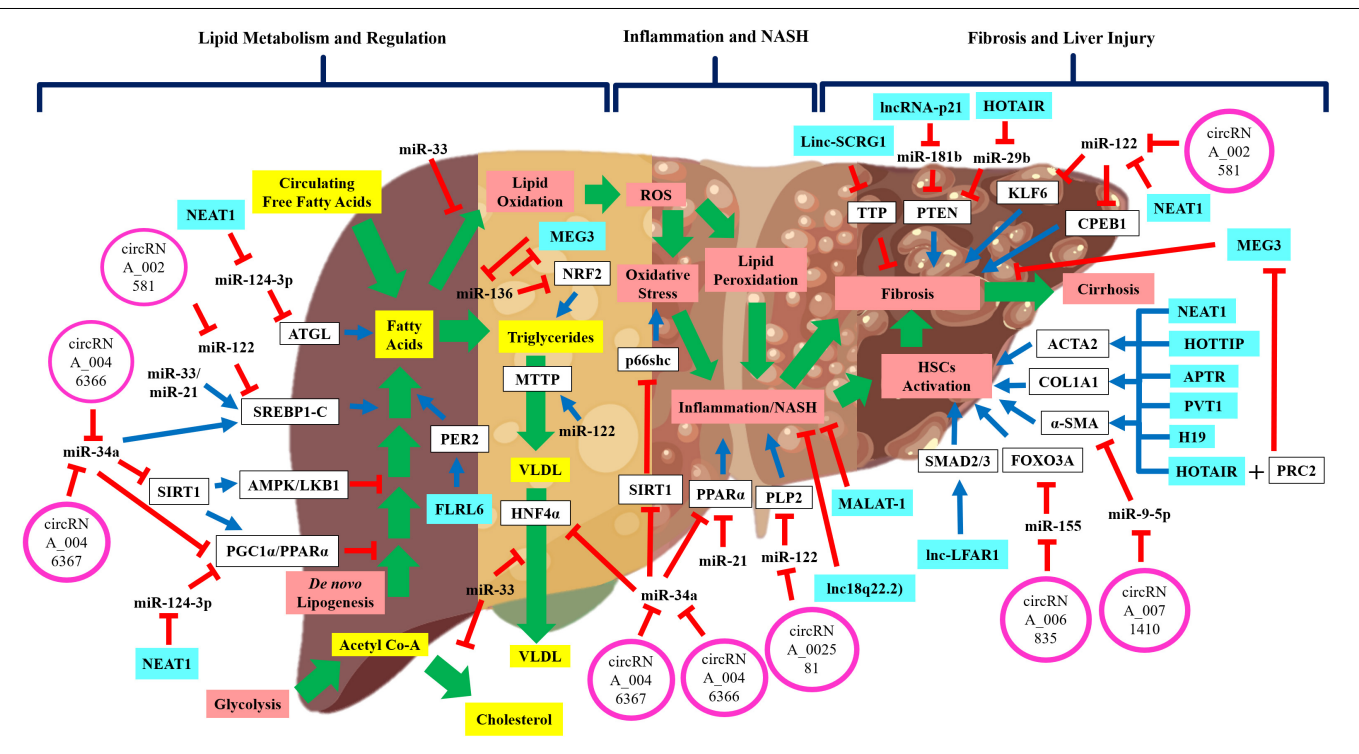

FIGURE 1 | The schematic diagram shows the regulatory non-coding RNAs in NAFLD disease development and progression. Wide-range of pathways and processes are regulated by non-coding RNAs in NAFLD development and progression. Emerging evidence shows that these regulatory non-coding RNAs interact with each other thus implying the complex regulation of NAFLD development and progression. Green arrow: pathway progression, Blue arrow: positive regulation, red inhibition arrow: negative regulation, and plus sign: combined action.

NAFLD is miR-21, which was up-regulated in the serum of NASH patients (Becker et al., 2015), but downregulation of miR21 expression resulted in reduced hepatic inflammation (Loyer et al., 2016). It is important to note that miR-21 is mainly expressed by the inflammatory and biliary cells, not hepatocytes (Loyer et al., 2016), thus the exact mechanism of how miR21 is involved in NASH remained unknown. Previous studies also have shown that miR-33 family members are the critical regulators of lipid metabolism and transport (Rayner et al., 2010; Dávalos et al., 2011) and their expressions were increased in NASH patients (Joel et al., 2016). A recent exploratory study using the aerobic exercise training as a therapy to reduce lipid levels in mice, had shown that the lipid-lowering effects observed were through miR-33 dependent autophagy (Ghareghani et al., 2018), thus confirming the regulatory role of miR-33 in lipid metabolism.

\section{MicroRNAs in Hepatic Fibrosis and Cirrhosis}

Hepatocyte-specific miR-122 expression was shown to be elevated in the serum or blood of the animal models of liver damage (Tryndyak et al., 2012; Clarke et al., 2014; Pirola et al., 2015; Yamada et al., 2015) and human NAFLD patients (Cermelli et al., 2011; Yamada et al., 2013; Celikbilek et al., 2014; Ward et al., 2014; Becker et al., 2015; Akuta et al., 2016; Salvoza et al., 2016). In contrast, the expression of miR-122 was found to be reduced in the liver tissues of NAFLD animal models and NAFLD patients (Cheung et al., 2008; Alisi et al., 2010; Tryndyak et al., 2012; Pirola et al., 2015; Aitana et al., 2016; Latorre et al., 2017). The reason for this discrepancy might be due to miR-122 is secreted out from damaged hepatocytes, and this was supported by the evidence of miR-122 localization that was mainly at the boundary of cell wall rather than in the cytoplasm ( $\mathrm{Su}$ et al., 2018). As miR-122 is exported out regularly into the circulation,
miR-122 possesses vast potential as a biomarker for liver injury and fibrosis progression.

Besides miR-122, inhibition of miR-21 resulted in reduced liver fibrosis and since miR-21 is mainly expressed by the inflammatory and biliary cells (Loyer et al., 2016), thus miR21 may play a significant role in cross-talk between liver microenvironment and hepatocytes, though further study is needed to determine this relationship. On the other hand, overexpression of miR-29b was also shown to inhibit liver fibrosis via COLIA1 and collagens in HSCs (Ogawa et al., 2012). In fact, the expression of the whole miR-29 family was downregulated in mice with liver fibrosis (Roy et al., 2015) and miR-29c expression was reduced in the liver of NASH patients (Aitana et al., 2016). Closer inspection on these molecules revealed that miR-29b is the leading player of hepatic fibrosis, where it could regulate several pro-fibrotic genes, including COLIA1, $\mathrm{SHH}$, and $c M Y C$ genes and the introduction of miR-29b managed to reduce collagen disposition with improvements in liver health (Kumar et al., 2016). Although these continuous updates on miRNAs involvement in NAFLD/NASH are enlightening, the practicality of using these miRNAs as therapeutic targets requires further assessments. The reason is that many of these miRNAs regulate multiple targets simultaneously and thus may unintendedly affect other important pathways. Therefore, most current studies on miRNAs are moving forward into looking at the efficacy, pharmacokinetics and pharmacodynamics of miRNA-based therapy to stop and cure the disease progression (Chakraborty et al., 2017).

\section{Long Non-coding RNA (IncRNAs)}

Long non-coding RNAs (lncRNAs) are RNA transcripts (>200 nucleotides in size) without the protein translation capacity and 
accounted for the majority of ncRNAs (Quinn and Chang, 2015). LncRNAs are transcribed from intergenic, exonic or the distal protein-coding regions by the RNA polymerase II and capped at $5^{\prime}$-end and polyadenylated at $3^{\prime}$-end (Quinn and Chang, 2015). Due to their locations are mainly in the poorly conserved regions of the genome, lncRNAs are difficult to characterize and highly diverse in their lengths, localizations and functions ( $\mathrm{Ma}$ et al., 2013; Quinn and Chang, 2015). Furthermore, they can form secondary and tertiary structures to interact with other molecules (Dhanoa et al., 2018), thus adding more to their heterogeneity and diversity. Generally, lncRNAs are grouped based on their structure, function, localization and interaction with other molecules (Dhanoa et al., 2018). Currently, there are five classes of lncRNAs: (1) sense lncRNAs, (2) antisense lncRNAs, (3) bidirectional lncRNAs, (4) intronic lncRNAs and (5) intergenic lncRNAs (Dhanoa et al., 2018). In terms of their functions, there are a few that have been identified: (1) as a decoy that binds a targeted protein or miRNA to suppress their functions, (2) as a guide by binding to the proteins and directs their localization, (3) as a scaffold by acting as a platform for the intermolecular interactions between proteins and RNAs, and (4) as a signal by interacting with transcription factors or chromatinmodifying enzymes to regulate gene expression ( $\mathrm{Ma}$ et al., 2013; Wong et al., 2018). On average, most lncRNAs have low expression though in some tissues or at specific developmental stages of the disease, elevated expression of lncRNAs can be observed (Ward et al., 2015), indicating the regulatory or specific role of lncRNA in disease development.

\section{LncRNAs in Hepatic Lipid Regulation, Steatosis and NASH}

The role of lncRNAs in liver disease is still not fully understood. Previous reviews of lncRNAs in liver disease (Takahashi et al., 2014), fibrosis (Jiang and Zhang, 2017; Hanson et al., 2018) and HCC (Qiu et al., 2017) have proposed that lncRNAs play an important role in the pathogenesis of liver diseases. Among these known lncRNAs, H19 was the first to be discovered and associated with liver disease (Ariel et al., 1998). Following that, many other lncRNAs have been identified to be involved in liver disease, particularly in HCC progression, including MEG3 (Braconi et al., 2011a), HULC (Panzitt et al., 2007), MALAT-1 (Lai et al., 2012), HOTAIR (Geng et al., 2011), TUC338 (Braconi et al., 2011b) and others (Takahashi et al., 2014). Despite what has been reported, the exact regulatory roles of lncRNAs in liver disease and NAFLD pathogenesis are still unknown.

Long non-coding RNAs are shown to regulate hepatic lipid regulation and metabolism (Figure 1). A small study of NAFLD patients $(n=5)$ has profiled the lncRNAs expression in the liver tissues and found that 535 lncRNAs had increased expression, and 1200 lncRNAs had reduced expression (Sun et al., 2015). These dysregulated lncRNAs were further investigated in silico for their functional and regulatory roles, in which many of these lncRNAs are involved in small molecule metabolic processes and regulation of cytoplasmic and endoplasmic reticulum parts (Sun et al., 2015). Profiling of lncRNAs in animal models of NAFLD revealed more information about lncRNAs' involvement in NAFLD. A study of NAFLD mice induced by high-fat diets showed that a total of 111 lncRNAs had increased expression, and 180 lncRNAs had reduced expression compared to the normal controls (Chen et al., 2017b). In this study (Chen et al., 2017b), seven IncRNAs were identified to be involved in the regulation of circadian rhythm. Upregulation of the lncRNA FLRL6 expression was shown to positively regulate PER2 expression, a regulator of circadian rhythm (Chen et al., 2017b). Participation of lncRNA FLRL6 in the regulation of PER2 expression may be important in NAFLD progression, as PER2 can regulate hepatic lipid metabolism via PPAR $\gamma$ (Grimaldi et al., 2010). Another identified lncRNA is FLRL2, in which its expression was reduced in NAFLD mice and the expression of its protein-coding gene, ARNT1, was also reduced (Chen et al., 2017b). Reduced ARNT1 expression was implicated in those with obesity (Paschos et al., 2012), high level of circulating fatty acids and ectopic fat formation in the liver (Shimba et al., 2011) suggesting there is a possible role of FLRL2 in hepatic lipid accumulation. Apart from that, two other lncRNAs were also identified to be involved in NAFLD namely, lncSTR that was shown to regulate systemic lipid metabolism (Li et al., 2015) and lncARS that was shown to regulate the fatty acid synthesis and oxidation via Akt/SREBP-1c pathway (Zhang et al., 2018). Identification of these dysregulated lncRNAs in hepatic lipid regulation may suggest that the expression of these lncRNAs can be used to identify the early stage of NAFLD.

\section{LncRNAs in Hepatic Fibrosis and Injury}

In terms of hepatic fibrosis, a recent and larger study of lncRNAs profiling in NASH patients $(n=48)$ identified a liver-specific pro-fibrotic lncRNA (lnc18q22.2) which was highly expressed in NASH patients, and this lncRNA expression was associated with greater NASH/NAFLD score and liver fibrosis index (Atanasovska et al., 2017). Similarly, another study of lncRNA profiling in the liver biopsies of NAFLD patients $(n=24)$ identified MALAT-1 as the potential regulator of inflammation and fibrosis (Leti et al., 2017). Investigation of hepatic tissue expression among fibrosis patients discovered more lncRNAs that were associated with fibrosis such as the HOXA distal transcript antisense RNA (HOTTIP) lncRNA that was elevated in fibrosis patients and even more so in cirrhosis patients ( $\mathrm{Li}$ et al., 2018), thus implying that HOTTIP expression may indeed represent hepatic injury severity. Another reported lncRNA is linc-SCRG1 that was upregulated in human cirrhotic tissues and its inhibition led to a reduction of fibrosis-related genes and apoptosis (Wu et al., 2019). The mechanism on how lincSCRG1 promotes fibrosis was shown to be via the inhibition of tristetraprolin (TTP) expression, which is a RNA-binding protein that is involved in the degradation of proteins (Wu et al., 2019).

Apart from human studies, animal models of liver fibrosis or injury also revealed more pro-fibrotic lncRNAs. The genomewide profiling in animal models of liver fibrosis found a liver fibrosis-associated lncRNA1 (lnc-LFAR1) that was upregulated in HSCs and its direct binding to $S$ mad2/3 proteins promoted TGF $\beta$ and Notch pathway activation and hepatocyte apoptosis (Zhang et al., 2017). Besides, a few other lncRNAs were also identified including the Alu-Mediated p21 Transcriptional Regulator (APTR) (Yu et al., 2015b), Plasmacytoma Variant Translocation 1 (PVT1) (Zheng et al., 2016), Homeobox (HOX) Transcript 
Antisense RNA (HOTAIR) (Bian et al., 2017), LncRNA H19 (Zhu et al.) and Nuclear Enriched Abundant Transcript 1 (NEAT1) (Yu et al., 2017b), in which all of these lncRNAs were upregulated in fibrotic tissues and activated HSCs. Most of these lncRNAs exerted their effects via ACTA2 and collagen, type 1, alpha 1 (COL1A1) proteins. With the identification of these pro-fibrotic lncRNAs, they can be used as biomarkers for fibrosis progression and the inhibition of their expression can be utilized as a treatment strategy.

As for lncRNAs with an anti-fibrotic role in the liver, genomewide lncRNAs profiling in the rat model of liver fibrosis had identified 231 differentially expressed $\ln c$ RNAs and characterized one lncRNA, namely NR_002155.1 that was able to suppress HSCs activation as it was significantly reduced in fibrotic liver (Gong et al., 2018). Other studies have also identified three other lncRNAs such as, MEG3 (Yu et al., 2018), GM5091 (Zhou B. et al., 2018) and lincRNA-p21 (Zheng et al., 2015), in which their expressions were reduced in fibrotic animals. Restoration of MEG3 and GM5091 expression caused downregulation of $\alpha-S M A$ and COL1A1 expression (Yu et al., 2018; Zhou B. et al., 2018). Similar to miRNAs and other conventional biomarkers, high false-positive and false-negative detections were also observed in these lncRNAs studies. Further research is required to fully characterize these lncRNAs particularly about their functional targets and their secretion into circulation.

\section{Co-regulatory Network Between IncRNAs and miRNAs}

One of the important findings in lncRNA studies is the role of lncRNA as a miRNA sponge and can prevent their actions toward the target mRNAs. This co-regulatory network between lncRNA and miRNA may indeed unravel the underlying mechanisms of NAFLD pathogenesis, though there are limited evidence available to support this molecular network (Figure 1). In the regulation of hepatic lipid, one study showed that IncRNA, NEAT1 increased adipose triglyceride lipase (ATGL) expression by competitive binding to miR124-3p and consequently increased levels of diacylglycerol and FFAs (Liu et al., 2018). This interaction between NEAT1/miR-124-3p disrupted the lipolysis and increased fatty acid oxidation via PPAR $\alpha$ signaling in liver cells (Liu et al., 2018). Another study also demonstrated that miR136 could suppress MEG3 and nuclear factor erythroid 2-related factor 2 (NRF2) expression and causing increased of TG contents and hepatocytes deaths (Wang and Wang, 2018). Due to these limited findings on the co-regulatory network between IncRNAs and miRNAs to control hepatic lipid regulation and metabolism, further work is needed to confirm these previous findings.

In contrast, there are more studies done to investigate the co-regulatory network between IncRNA and other molecules in hepatic liver fibrosis or injury. One such interaction is the relationship between IncRNA, NEAT1 and miR-122 in the regulation of liver fibrosis (Yu et al., 2017b). In this animal model of fibrosis (Yu et al., 2017b), it was demonstrated that miR-122 reduced the Kruppel-like factor 6 (KLF6) expression and NEAT1 inhibited the miR-122 regulatory action via competitive binding to miR-122. Suppression of miR-122 by NEAT1 caused increased of KLF6 expression in HSCs and promoted fibrogenesis (Yu et al., 2017b), thus confirming the coregulatory network of NEAT1/miR-122/KLF6 signaling cascade in fibrosis. Similarly, in other studies of fibrotic animals and HSCs, other co-regulatory networks were identified including, the HOTAIR/miR-29b/PTEN cascade (Yu et al., 2017a), lincp21/miR-181b/PTEN cascade (Yu et al., 2016), PVT1/miR152/PTCH1 signaling (Zheng et al., 2016), H19/miR-148a/USP4 and TGF- $\beta$ pathway (Zhu J. et al., 2018), MALAT1/miR101b/RAS-related C3 botulinum substrate 1 (RAC1) (Yu et al., 2015a), HOTTIP/miR-148a/TGF- $\beta$ receptors (Li et al., 2018), HOTAIR/miR-148b/DNMT1 (Bian et al., 2017) and MEG3/miR-212/Smoothened (SMO) signaling (Yu et al., 2018). Interestingly, in one study of fibrotic animals, IncRNA HOTAIR mediated suppression of another lncRNA, MEG3 via epigenetic mechanisms (Bian et al., 2017). It was shown that HOTAIR acted as a platform or guide for binding of polycomb repressive complex 2 (PRC2) to the promoter region of MEG3 gene and induced histone H3K27me3 suppression (Bian et al., 2017). This promoter modifications then led to an inhibition of MEG3 expression and promoted liver fibrosis (Bian et al., 2017). This evidence of lncRNA-lncRNA interaction adds more complexity to the known molecular regulatory network, especially in liver fibrosis.

\section{Circular RNAs (circRNAs)}

Circular RNAs (circRNAs) are the structurally covalent loop RNAs without a 5'-cap and 3'-tail (Yao et al., 2017). Previous studies have shown that circRNAs are the products of backsplicing events, which allows the formation of the loop structure (Wilusz Jeremy, 2018). This circular structure provides greater stability for this RNA species due to the protection against degradation by the exoribonucleases. With advancement in RNA sequencing analysis and in-depth molecular research, circRNAs are shown to regulate the gene expression at transcription and post-transcription levels by acting as a miRNA sponge as well as interacting with lncRNAs, mRNA and proteins (Wilusz Jeremy, 2018). Research in circRNAs has garnered much interest recently due to the circRNAs stability in the circulation and their potential to serve as biomarkers or therapeutic targets (Abu and Jamal, 2016; Wilusz Jeremy, 2018). Majority of the current studies on circRNAs in liver diseases are focused on HCC and hepatitis (Yao et al., 2017), with limited information available for NAFLD development and progression. Due to this limitation, we discussed the circRNAs role in NAFLD by exploring the coregulatory network between circRNAs and their miRNA targets as well as their downstream molecular targets (Figure 1).

\section{CircRNAs in Hepatic Lipid Regulation, Steatosis and NASH}

The regulatory network between circRNAs and miRNAs to control gene expression has emerged as a new understanding of the molecular regulation in disease development (Figure 1). A study performed by Jin et al. (2016) profiled circRNAs expression from liver tissues of NASH mice and they found that 69 circRNAs had increased expression, and 63 circRNAs had reduced expression (Jin et al., 2016). Among these circRNAs, three of them have potential interaction with miR-122 as its 
sponge, thus affecting their respective genes in NASH (Jin et al., 2016). These include circRNA_002581 interaction with miR-122 and its respective gene of SLC1A5, circRNA_002581 interaction with miR-122 and its respective gene PLP2 and circRNA_002581 interaction with miR-122 and its respective gene, CPEB1 (Jin et al., 2016). Identification of this circRNA/miRNA/mRNA interaction thus establishes the role of circRNAs in NASH and possibly in NAFLD.

Another exploratory circRNAs profiling experiment was performed in HepG2 cells with hepatic steatosis induced by a high-fat stimulation (Guo et al., 2017b). A total of 357 circRNAs were found to be dysregulated with a reduced expression of circRNA_021412 and confirmation of co-regulation of LPIN1 expression by circRNA_021412 and miR-1972 (Guo et al., 2017b). Another study pursued an in-depth molecular analysis of circRNA_0046367 expression and function in NAFLD. Expression of circRNA_0046367 was reduced in hepatic steatosis resulting in an increased expression of miR-34a (Guo et al., 2017a). miR-34a was shown to reduce PPAR $\alpha$ expression, and the presence of circRNA_0046367 inhibited this miR34a suppression of PPAR $\alpha$ expression, in conjunction with evidence of improved mitochondrial function and prevention of hepatotoxicity (Guo et al., 2017a). This co-regulation of PPAR $\alpha$ expression by circRNA and miR-34a was replicated again by the same researchers but using a different circRNA, circRNA_0046366 that also showed a prevention of hepatic steatosis (Guo et al., 2018). Although only a few studies have embarked on circRNAs research in NAFLD, this identification and the proof-of-concept study to elucidate the co-regulatory network of circRNA-miRNA involved in hepatic lipid regulation, have opened a new window of understanding the molecular regulation underlying the early stage of NAFLD pathogenesis.

\section{CircRNAs in Hepatic Fibrosis and Cirrhosis}

Studies of circRNAs in hepatic fibrosis have identified few circRNAs that are associated with fibrosis or liver injury. A microarray profiling of fibrotic HSCs induced by irradiation showed that 179 circRNAs expression were upregulated and 630 circRNAs expression were downregulated (Chen et al., 2017c). In this study (Chen et al., 2017c), they also investigated the role of circ_0071410 in HSCs activation and found that circ_0074410 reduced miR-9-5p expression and promoted HSC activation via $\alpha$-SMA protein. Similarly, a global microarray profiling of circRNAs in the animal model of fibrosis revealed that 69 circRNAs were differentially expressed in the fibrotic liver tissues, with 14 of them had increased expression, and 55 had reduced expression (Zhou Y. et al., 2018). Among these circRNAs, circRNA_34116 was shown to inhibit HSCs activation possibly via miR-22-3p and its targets, BMP7 as predicted by the bioinformatics analysis (Zhou Y. et al., 2018). Another study of HSC inhibition by thymosin beta 4 treatment has identified 644 circRNAs that were differentially expressed and circRNA_0067835 expression was significantly increased in activated HSCs (Zhu L. et al., 2018). It was also shown that circRNA_0067835 acted as a miR-155 sponge and elevated the FOXO3a expression thus promoted HSCs activation and fibrosis (Zhu
L. et al., 2018). Despite these limited findings of circRNAs roles in liver fibrosis, the evidence of their regulatory interaction with miRNAs and their target mRNAs showed that understanding the molecular regulation and mechanisms underlying liver fibrosis could potentially discover a novel therapeutic target.

\section{Other Emerging Regulatory ncRNAs}

Besides the well-established ncRNAs, the P-element-induced wimpy testis (PIWI)-interacting RNAs (piRNAs) has garnered new interest as the new small regulatory ncRNAs. The piRNAs are similar in length to miRNAs ( 26-30 nucleotides) and have the 2'-O-methylation at the 3'-end (Weng et al., 2019). Unlike miRNAs, piRNAs precursors are single-stranded transcripts without any secondary structures and these precursors are generated from the specific genomic regions with repetitive elements (Weng et al., 2019). The precursors piRNAs are then modified post-transcriptionally (2'-O-methylation) to produce mature piRNAs and loaded onto PIWI family proteins to form silencing complexes to repress transposable elements (TEs), at both transcriptional and post-transcriptional levels in germline cells (Iwasaki et al., 2015; Sarkar et al., 2017). The presence of TEs in the human genome is high with up to $50 \%$ from overall genome components. However, the majority of these TEs are silenced (International Human Genome Sequencing Consortium, 2001; de Koning et al., 2011; Wang and Jordan, 2018) to prevent their notorious effects of causing genome instability via chromosomal breakages, insertions and genomic rearrangements (Wang and Jordan, 2018). Thus, piRNA is known as the protector of genome integrity (Iwasaki et al., 2015; Sarkar et al., 2017) due to their suppression of TEs. Previous studies showed that piRNAs can regulate mRNA and lncRNAs expression, in which piRNAs are implicated in mitochondrial surface lipid signaling (Huang et al., 2011; Shiromoto et al., 2013). However, to which extent these piRNAs are involved in NAFLD development and progression remain unknown, thus requires further investigation to evaluate these piRNAs in the regulation of lipid metabolism and signaling.

Another ncRNA that has been on the spotlight recently is the enhancer RNAs (eRNAs) (De Santa et al., 2010; Kim et al., 2010; Djebali et al., 2012) that usually are $0.5-5 \mathrm{~kb}$ in size and act as a transcription regulator via the enhancer molecules (Chen et al., 2017a; Azofeifa et al., 2018). The exact mechanism by which the eRNAs regulate the gene expression is still unknown. Studies have shown that eRNAs behave similarly to lncRNAs, in which they both regulate or promote the expression of nearby genes (Li et al., 2013; Melo et al., 2013), mainly via the promoter or enhancer regions (Bose and Berger, 2017; Meng and Bartholomew, 2017; Jiao et al., 2018). Limited information is available on the regulatory role of eRNAs in disease and much less for NAFLD. Interestingly, a recent study showed that eRNAs with $\mathrm{m}^{5} \mathrm{C}$ mark were associated with metabolic stress in vitro and in vivo; specifically, these eRNAs interacted with PGC1 $\alpha$ to regulate NSUN7 and SIRT1 gene expression (Aguilo et al., 2016). This finding suggests that eRNAs may be involved in NAFLD progression, as both PGCl $\alpha$ and SIRT1 are implicated in fatty acid oxidation and NAFLD development (Rodgers et al., 2005; Bellanti et al., 2014; Choi et al., 2017). 


\section{REGULATORY ncRNAs: CHALLENGES AND FUTURE DIRECTION}

Among the ncRNAs in NAFLD, miRNAs is the most well-defined and have been extensively studied (Baffy, 2015; Gerhard and DiStefano, 2015; DiStefano and Gerhard, 2016; He et al., 2016; Otsuka et al., 2016; Lin et al., 2017). Emerging reports on other regulatory ncRNAs, including lncRNAs, circRNAs, piRNAs, and eRNAs have highlighted that co-regulation and interactions between these ncRNAs may expand the current understanding of the molecular regulation and its complexity in disease development and progression. The co-regulatory networks of circRNAs, lncRNAs, and miRNAs in NAFLD pathophysiology offer new possibilities in finding the biomarkers and therapeutic targets. Identification of non-invasive and sensitive biomarkers for NASH and fibrosis is crucial as the current liver biopsy method to assess NASH/fibrosis can cause further complication to liver health and the imaging methods can only detect steatosis but not NASH (Wruck et al., 2017). To date, many of these studies remain in preliminary stages with most of the works are done focusing on profiling and identifying their expressions in correlation to the NAFLD stages. More studies are needed to elucidate the mechanisms of how these ncRNAs regulate NAFLD progression and which molecules they interact with, especially

\section{REFERENCES}

Abu, N., and Jamal, R. (2016). Circular RNAs as promising biomarkers: a minireview. Front. Physiol. 7:355. doi: 10.3389/fphys.2016.00355

Aguilo, F., Li, S., Balasubramaniyan, N., Sancho, A., Benko, S., Zhang, F., et al. (2016). Deposition of 5-methylcytosine on enhancer RNAs enables the coactivator function of PGC-1 $\alpha$. Cell Rep. 14, 479-492. doi: 10.1016/j.celrep. 2015.12.043

Aitana, B. B., Josep, M. A., Pilar, M., Arnau, A. M., Moisés, B. M., Diana, D., et al. (2016). Deregulated hepatic microRNAs underlie the association between non-alcoholic fatty liver disease and coronary artery disease. Liver Int. 36, 1221-1229. doi: 10.1111/liv.13097

Akuta, N., Kawamura, Y., Suzuki, F., Saitoh, S., Arase, Y., Fujiyama, S., et al. (2016). Analysis of association between circulating miR-122 and histopathological features of nonalcoholic fatty liver disease in patients free of hepatocellular carcinoma. BMC Gastroenterol. 16:141. doi: 10.1186/s12876-016-0557-6

Alisi, A., Da Sacco, L., Bruscalupi, G., Piemonte, F., Panera, N., De Vito, R., et al. (2010). Mirnome analysis reveals novel molecular determinants in the pathogenesis of diet-induced nonalcoholic fatty liver disease. Lab. Invest. 91:283. doi: 10.1038/labinvest.2010.166

Ananthanarayanan, A. T. (2018). Role of mitochondria and mitochondria-targeted agents in non-alcoholic fatty liver disease. Clin. Exp. Pharmacol. Physiol. 45, 413-421. doi: 10.1111/1440-1681.12886

Araújo, A. R., Rosso, N., Bedogni, G., Tiribelli, C., and Bellentani, S. (2018). Global epidemiology of non-alcoholic fatty liver disease/non-alcoholic steatohepatitis: what we need in the future. Liver Int. 38, 47-51. doi: 10.1111/liv.13643

Ariel, I., Miao, H. Q., Ji, X. R., Schneider, T., Roll, D., de Groot, N., et al. (1998) Imprinted $\mathrm{H} 19$ oncofetal RNA is a candidate tumour marker for hepatocellular carcinoma. Mol. Pathol. 51, 21-25. doi: 10.1136/mp.51.1.21

Atanasovska, B., Rensen, S. S., Sijde, M. R., Marsman, G., Kumar, V., Jonkers, I., et al. (2017). A liver-specific long noncoding RNA with a role in cell viability is elevated in human nonalcoholic steatohepatitis. Hepatology 66, 794-808. doi: 10.1002/hep.29034

Azofeifa, J. G., Allen, M. A., Hendrix, J. R., Read, T., Rubin, J. D., and Dowell, R. D. (2018). Enhancer RNA profiling predicts transcription factor activity. Genome Res. 28, 334-344. doi: 10.1101/gr.225755.117 in regards to lncRNAs that have diverse biological functions (Takahashi et al., 2014). Unlike miRNAs, lncRNAs are poorly conserved between species, thus to experimentally demonstrate their functions in in vivo studies is challenging. Similarly, research of circRNAs in NAFLD is still in its infancy but their ability to control miRNAs and their stability in the circulating biofluids are making them as the most promising biomarker and therapeutic agent. Therefore, in-depth understanding of these ncRNAs in NAFLD pathogenesis and their efficiency and specificity as biomarkers or therapeutic targets are the unmet need for better NAFLD outcomes and disease management.

\section{AUTHOR CONTRIBUTIONS}

SAS drafted and wrote the manuscript. NM wrote the manuscript and critically reviewed the manuscript. RJ critically advised and reviewed the manuscript.

\section{FUNDING}

This work was funded by the UKM Young Lecture Incentive Grant (GGPM) (Grant No: GGPM-2017-017).

Baffy, G. (2015). MicroRNAs in nonalcoholic fatty liver disease. J. Clin. Med. 4, 1977-1988. doi: 10.3390/jcm4121953

Becker, P. P., Rau, M., Schmitt, J., Malsch, C., Hammer, C., Bantel, H., et al. (2015). Performance of serum microRNAs $-122,-192$ and -21 as biomarkers in patients with non-alcoholic steatohepatitis. PLoS One 10:e0142661. doi: 10.1371/journal. pone. 0142661

Bedogni, G., Miglioli, L., Masutti, F., Tiribelli, C., Marchesini, G., and Bellentani, S. (2005). Prevalence of and risk factors for nonalcoholic fatty liver disease: the dionysos nutrition and liver study. Hepatology 42, 44-52. doi: 10.1002/hep. 20734

Bellanti, F., Mitarotonda, D., Tamborra, R., Blonda, M., Iannelli, G., Petrella, A., et al. (2014). OP2-6 - Oxysterols induce mitochondrial impairment and hepatocellular toxicity in non-alcoholic fatty liver disease. Free Radic. Biol. Med. 75, S16-S17. doi: 10.1016/j.freeradbiomed.2014.10.594

Bian, E.-B., Wang, Y.-Y., Yang, Y., Wu, B.-M., Xu, T., Meng, X.-M., et al. (2017). Hotair facilitates hepatic stellate cells activation and fibrogenesis in the liver. Biochim. Biophys. Acta 1863, 674-686. doi: 10.1016/j.bbadis.2016.12.009

Bose, D. A., and Berger, S. L. (2017). eRNA binding produces tailored CBP activity profiles to regulate gene expression. RNA Biol. 14, 1655-1659. doi: 10.1080/ 15476286.2017.1353862

Braconi, C., Kogure, T., Valeri, N., Huang, N., Nuovo, G., Costinean, S., et al. (2011a). microRNA-29 can regulate expression of the long non-coding RNA gene MEG3 in hepatocellular cancer. Oncogene 30, 4750-4756. doi: 10.1038/ onc. 2011.193

Braconi, C., Valeri, N., Kogure, T., Gasparini, P., Huang, N., Nuovo, G. J., et al. (2011b). Expression and functional role of a transcribed noncoding RNA with an ultraconserved element in hepatocellular carcinoma. Proc. Natl. Acad. Sci. U.S.A. 108, 786-791. doi: 10.1073/pnas.1011098108

Brandt, S., Roos, J., Inzaghi, E., Kotnik, P., Kovac, J., Battelino, T., et al. (2018). Circulating levels of miR-122 and nonalcoholic fatty liver disease in prepubertal obese children. Pediatr. Obesity 13, 175-182. doi: 10.1111/ijpo.12261

Castro, R. E., Ferreira, D. M. S., Afonso, M. B., Borralho, P. M., Machado, M. V., Cortez-Pinto, H., et al. (2013). miR-34a/SIRT1/p53 is suppressed by ursodeoxycholic acid in the rat liver and activated by disease severity in human non-alcoholic fatty liver disease. J. Hepatol. 58, 119-125. doi: 10.1016/j.jhep. 2012.08.008 
Celikbilek, M., Baskol, M., Taheri, S., Deniz, K., Dogan, S., Zararsiz, G., et al. (2014). Circulating microRNAs in patients with non-alcoholic fatty liver disease. World J. Hepatol. 6, 613-620. doi: 10.4254/wjh.v6.i8.613

Cermelli, S., Ruggieri, A., Marrero, J. A., Ioannou, G. N., and Beretta, L. (2011). Circulating MicroRNAs in patients with chronic hepatitis c and non-alcoholic fatty liver disease. PLoS One 6:e23937. doi: 10.1371/journal.pone.0023937

Chakraborty, C., Sharma, A. R., Sharma, G., Doss, C. G. P., and Lee, S.-S. (2017). Therapeutic miRNA and siRNA: moving from bench to clinic as next generation medicine. Mol. Ther. 8, 132-143. doi: 10.1016/j.omtn.2017. 06.005

Chang, T.-C., Wentzel, E. A., Kent, O. A., Ramachandran, K., Mullendore, M., Lee, K. H., et al. (2007). Transactivation of miR-34a by p53 broadly influences gene expression and promotes apoptosis. Mol. Cell 26, 745-752. doi: 10.1016/j. molcel.2007.05.010

Chen, H., Du, G., Song, X., and Li, L. (2017a). Non-coding transcripts from enhancers: new insights into enhancer activity and gene expression regulation. Genom. Proteom. Bioinform. 15, 201-207. doi: 10.1016/j.gpb.2017.02.003

Chen, Y., Huang, H., Xu, C., Yu, C., and Li, Y. (2017b). Long non-coding RNA profiling in a non-alcoholic fatty liver disease rodent model: new insight into pathogenesis. Int. J. Mol. Sci. 18:21. doi: 10.3390/ijms18010021

Chen, Y., Yuan, B., Wu, Z., Dong, Y., Zhang, L., and Zeng, Z. (2017c). Microarray profiling of circular RNAs and the potential regulatory role of hsa_circ_0071410 in the activated human hepatic stellate cell induced by irradiation. Gene 629, 35-42. doi: 10.1016/j.gene.2017.07.078

Cheung, O., Puri, P., Eicken, C., Contos, M. J., Mirshahi, F., Maher, J. W., et al. (2008). Nonalcoholic steatohepatitis is associated with altered hepatic MicroRNA expression. Hepatology 48, 1810-1820. doi: 10.1002/hep.22569

Choi, S. E., Kwon, S., Seok, S., Xiao, Z., Lee, K.-W., Kang, Y., et al. (2017). Obesity-linked phosphorylation of SIRT1 by casein kinase 2 inhibits its nuclear localization and promotes fatty liver. Mol. Cell. Biol. 37, e00006-e00017. doi: 10.1128/MCB.00006-17

Clarke, J. D., Sharapova, T., Lake, A. D., Blomme, E., Maher, J., and Cherrington, N. J. (2014). Circulating microRNA 122 in the methionine and choline deficient mouse model of nonalcoholic steatohepatitis. J. Appl. Toxicol. 34, 726-732. doi: $10.1002 /$ jat. 2960

Csak, T., Bala, S., Lippai, D., Satishchandran, A., Catalano, D., Kodys, K., et al. (2015). microRNA-122 regulates hypoxia-inducible factor-1 and vimentin in hepatocytes and correlates with fibrosis in diet-induced steatohepatitis. Liver Int. 35, 532-541. doi: 10.1111/liv.12633

Dávalos, A., Goedeke, L., Smibert, P., Ramírez, C. M., Warrier, N. P., Andreo, U., et al. (2011). miR-33a/b contribute to the regulation of fatty acid metabolism and insulin signaling. Proc. Natl. Acad. Sci. U.S.A. 108, 9232-9237. doi: 10.1073/ pnas. 1102281108

de Koning, A. P. J., Gu, W., Castoe, T. A., Batzer, M. A., and Pollock, D. D. (2011). Repetitive elements may comprise over two-thirds of the human genome. PLoS Genet. 7:e1002384. doi: 10.1371/journal.pgen.1002384

De Santa, F., Barozzi, I., Mietton, F., Ghisletti, S., Polletti, S., Tusi, B. K., et al. (2010). A large fraction of extragenic RNA Pol II transcription sites overlap enhancers. PLoS Biol. 8:e1000384. doi: 10.1371/journal.pbio.1000384

Dhanoa, J. K., Sethi, R. S., Verma, R., Arora, J. S., and Mukhopadhyay, C. S. (2018). Long non-coding RNA: its evolutionary relics and biological implications in mammals: a review. J. Anim. Sci. Technol. 60, 25-25. doi: 10.1186/s40781-0180183-7

Ding, J., Li, M., Wan, X., Jin, X., Chen, S., Yu, C., et al. (2015). Effect of miR-34a in regulating steatosis by targeting PPAR $\alpha$ expression in nonalcoholic fatty liver disease. Sci. Rep. 5:13729. doi: 10.1038/srep13729

DiStefano, J. K., and Gerhard, G. S. (2016). Circulating microRNAs in nonalcoholic fatty liver disease. Expert Rev. Gastroenterol. Hepatol. 10, 161-163. doi: 10.1586/ 17474124.2016.1125290

Djebali, S., Davis, C. A., Merkel, A., Dobin, A., Lassmann, T., Mortazavi, A. M., et al. (2012). Landscape of transcription in human cells. Nature 489, 101-108. doi: $10.1038 /$ nature 11233

Elmén, J., Lindow, M., Schütz, S., Lawrence, M., Petri, A., Obad, S., et al. (2008). LNA-mediated microRNA silencing in non-human primates. Nature 452:896. doi: $10.1038 /$ nature06783

Esau, C., Davis, S., Murray, S. F., Yu, X. X., Pandey, S. K., Pear, M., et al. (2006). miR-122 regulation of lipid metabolism revealed by in vivo antisense targeting. Cell Metab. 3, 87-98. doi: 10.1016/j.cmet.2006.01.005
Eslam, M., Valenti, L., and Romeo, S. (2018). Genetics and epigenetics of NAFLD and NASH: clinical impact. J. Hepatol. 68, 268-279. doi: 10.1016/j.jhep.2017. 09.003

Friedman, S. L. (2008). Hepatic stellate cells: protean, multifunctional, and enigmatic cells of the liver. Physiol. Rev. 88, 125-172. doi: 10.1152/physrev. 00013.2007

Geng, Y., Xie, S., Li, Q., Ma, J., and Wang, G. (2011). Large intervening non-coding RNA HOTAIR is associated with hepatocellular carcinoma progression. J. Int. Med. Res. 39, 2119-2128. doi: 10.1177/147323001103900608

Gentile, C. L., and Pagliassotti, M. J. (2008). The role of fatty acids in the development and progression of nonalcoholic fatty liver disease. J. Nutr. Biochem. 19, 567-576. doi: 10.1016/j.jnutbio.2007.10.001

Gerhard, G. S., and DiStefano, J. K. (2015). Micro RNAs in the development of nonalcoholic fatty liver disease. World J. Hepatol. 7, 226-234. doi: 10.4254/wjh.v7. i2.226

Ghareghani, P., Shanaki, M., Ahmadi, S., Khoshdel, A. R., Rezvan, N., Meshkani, R., et al. (2018). Aerobic endurance training improves nonalcoholic fatty liver disease (NAFLD) features via miR-33 dependent autophagy induction in high fat diet fed mice. Obesity Res. Clin. Pract. 12, 80-89. doi: 10.1016/j.orcp.2017. 01.004

Gong, Z., Tang, J., Xiang, T., Lin, J., Deng, C., Peng, Y., et al. (2018). Genome-wide identification of long noncoding RNAs in CCl4-induced liver fibrosis via RNA sequencing. Mol. Med. Rep. 18, 299-307. doi: 10.3892/mmr.2018.8986

Grimaldi, B., Bellet, M. M., Katada, S., Astarita, G., Hirayama, J., Amin, R. H., et al. (2010). PER2 controls lipid metabolism by direct regulation of PPAR $\gamma$. Cell Metab. 12, 509-520. doi: 10.1016/j.cmet.2010.10.005

Guo, X.-Y., Chen, J.-N., Sun, F., Wang, Y.-Q., Pan, Q., and Fan, J.-G. (2017a). circRNA_0046367 prevents hepatoxicity of lipid peroxidation: an inhibitory role against hepatic steatosis. Oxid. Med. Cell. Longev. 2017:3960197. doi: 10. 1155/2017/3960197

Guo, X.-Y., He, C.-X., Wang, Y.-Q., Sun, C., Li, G.-M., Su, Q., et al. (2017b). Circular RNA profiling and bioinformatic modeling identify its regulatory role in hepatic steatosis. Biomed Res. Int. 2017:5936171. doi: 10.1155/2017/593 6171

Guo, X.-Y., Sun, F., Chen, J.-N., Wang, Y.-Q., Pan, Q., and Fan, J.-G. (2018). circRNA_0046366 inhibits hepatocellular steatosis by normalization of PPAR signaling. World J. Gastroenterol. 24, 323-337. doi: 10.3748/wjg.v24.i3.323

Hanson, A., Wilhelmsen, D., and DiStefano, J. K. (2018). The role of long non-coding RNAs (lncRNAs) in the development and progression of fibrosis associated with Nonalcoholic Fatty Liver Disease (NAFLD). Non-coding RNA 4:18. doi: $10.3390 /$ ncrna4030018

He, Z., Hu, C., and Jia, W. (2016). miRNAs in non-alcoholic fatty liver disease. Front. Med. 10:389-396. doi: 10.1007/s11684-016-0468-5

Hon, C.-C., Ramilowski, J. A., Harshbarger, J., Bertin, N., Rackham, O. J. L., Gough, J., et al. (2017). An atlas of human long non-coding RNAs with accurate 5' ends. Nature 543:199. doi: 10.1038/nature21374

Hsu, S. H., Wang, B., Kota, J., Yu, J., Costinean, S., Kutay, H., et al. (2012). Essential metabolic, anti-inflammatory, and anti-tumorigenic functions of miR-122 in liver. J. Clin. Invest. 122, 2871-2883. doi: 10.1172/JCI63539

Huang, H., Gao, Q., Peng, X., Choi, S.-Y., Sarma, K., Ren, H., et al. (2011). piRNAassociated germline nuage formation and spermatogenesis require MitoPLD profusogenic mitochondrial-surface lipid signaling. Dev. Cell 20, 376-387. doi: 10.1016/j.devcel.2011.01.004

Ibrahim, S. H., Hirsova, P., and Gores, G. J. (2018). Non-alcoholic steatohepatitis pathogenesis: sublethal hepatocyte injury as a driver of liver inflammation. Gut 67, 963-972. doi: 10.1136/gutjnl-2017-315691

International Human Genome Sequencing Consortium. (2001). Initial sequencing and analysis of the human genome. Nature 409:860. doi: 10.1038/35057062

Iwasaki, Y. W., Siomi, M. C., and Siomi, H. (2015). PIWI-interacting RNA: its biogenesis and functions. Annu. Rev. Biochem. 84, 405-433. doi: 10.1146/ annurev-biochem-060614-034258

Jiang, X., and Zhang, F. (2017). Long noncoding RNA: a new contributor and potential therapeutic target in fibrosis. Epigenomics 9, 1233-1241. doi: 10.2217/ epi-2017-0020

Jiao, W., Chen, Y., Song, H., Li, D., Mei, H., Yang, F., et al. (2018). HPSE enhancer RNA promotes cancer progression through driving chromatin looping and regulating hnRNPU/p300/EGR1/HPSE axis. Oncogene 37, 2728-2745. doi: $10.1038 / \mathrm{s} 41388-018-0128-0$ 
Jin, X., Feng, C. Y., Xiang, Z., Chen, Y. P., and Li, Y. M. (2016). CircRNA expression pattern and circRNA-miRNA-mRNA network in the pathogenesis of nonalcoholic steatohepatitis. Oncotarget 7, 66455-66467. doi: 10.18632/ oncotarget.12186

Joel, V. B., Roxana, G. V., Hugo, H. P., Hugo, V. R., Paola, L. M., Fausto, S. M., et al. (2016). Hepatic miR-33a/miR-144 and their target gene ABCA1 are associated with steatohepatitis in morbidly obese subjects. Liver Int. 36, 1383-1391. doi: 10.1111/liv.13109

Kim, T.-K., Hemberg, M., Gray, J. M., Costa, A. M., Bear, D. M., Wu, J., et al. (2010). Widespread transcription at neuronal activity-regulated enhancers. Nature 465, 182-187. doi: 10.1038/nature09033

Krützfeldt, J., Rajewsky, N., Braich, R., Rajeev, K. G., Tuschl, T., Manoharan, M., et al. (2005). Silencing of microRNAs in vivo with 'antagomirs'. Nature 438:685. doi: 10.1038 /nature04303

Kumar, V., Mondal, G., Dutta, R., and Mahato, R. I. (2016). Co-delivery of small molecule hedgehog inhibitor and miRNA for treating liver fibrosis. Biomaterials 76, 144-156. doi: 10.1016/j.biomaterials.2015.10.047

Lai, M. C., Yang, Z., Zhou, L., Zhu, Q. Q., Xie, H. Y., Zhang, F., et al. (2012). Long non-coding RNA MALAT-1 overexpression predicts tumor recurrence of hepatocellular carcinoma after liver transplantation. Med. Oncol. 29, 1810-1816. doi: 10.1007/s12032-011-0004-z

Latorre, J., Moreno-Navarrete, J. M., Mercader, J. M., Sabater, M., Rovira, Ò, Gironès, J., et al. (2017). Decreased lipid metabolism but increased FA biosynthesis are coupled with changes in liver microRNAs in obese subjects with NAFLD. Int. J. Obesity 41:620. doi: 10.1038/ijo.2017.21

Leti, F., Legendre, C., Still, C. D., Chu, X., Petrick, A., Gerhard, G. S., et al. (2017). Altered expression of MALAT1 lncRNA in nonalcoholic steatohepatitis fibrosis regulates CXCL5 in hepatic stellate cells. Trans. Res. 190:25-39.e21. doi: 10.1016/j.trsl.2017.09.001

Li, P., Ruan, X., Yang, L., Kiesewetter, K., Zhao, Y., Luo, H., et al. (2015). A liverenriched long non-coding RNA, IncLSTR, regulates systemic lipid metabolism in mice. Cell Metab. 21, 455-467. doi: 10.1016/j.cmet.2015.02.004

Li, W., Notani, D., Ma, Q., Tanasa, B., Nunez, E., Chen, A. Y., et al. (2013). Functional importance of eRNAs for estrogen-dependent transcriptional activation events. Nature 498, 516-520. doi: 10.1038/nature12210

Li, Z., Wang, J., Zeng, Q., Hu, C., Zhang, J., Wang, H., et al. (2018). Long noncoding RNA HOTTIP promotes mouse hepatic stellate cell activation via downregulating miR-148a. Cell. Physiol. Biochem. 51, 2814-2828. doi: 10.1159/ 000496012

Lin, H., Ewing, L. E., Koturbash, I., Gurley, B. J., and Miousse, I. R. (2017), MicroRNAs as biomarkers for liver injury: current knowledge, challenges and future prospects. Food Chem. Toxicol. 110, 229-239. doi: 10.1016/j.fct.2017. 10.026

Liu, X., Liang, Y., Song, R., Yang, G., Han, J., Lan, Y., et al. (2018). Long non-coding RNA NEAT1-modulated abnormal lipolysis via ATGL drives hepatocellular carcinoma proliferation. Mol. Cancer 17:90. doi: 10.1186/s12943-018-0838-5

Liu, X.-L., Pan, Q., Zhang, R.-N., Shen, F., Yan, S.-Y., Sun, C., et al. (2016). Diseasespecific miR-34a as diagnostic marker of non-alcoholic steatohepatitis in a Chinese population. World J. Gastroenterol. 22, 9844-9852. doi: 10.3748/wjg. v22.i44.9844

Loyer, X., Paradis, V., Hénique, C., Vion, A.-C., Colnot, N., Guerin, C. L., et al. (2016). Liver microRNA-21 is overexpressed in non-alcoholic steatohepatitis and contributes to the disease in experimental models by inhibiting PPAR $\alpha$ expression. Gut 65, 1882-1894. doi: 10.1136/gutjnl-2014-308883

Ma, L., Bajic, V. B., and Zhang, Z. (2013). On the classification of long non-coding RNAs. RNA Biol. 10, 924-933. doi: 10.4161/rna.24604

Magee, N., Zou, A., and Zhang, Y. (2016). Pathogenesis of nonalcoholic steatohepatitis: interactions between liver parenchymal and nonparenchymal cells. Biomed Res. Int. 2016: 5170402. doi: 10.1155/2016/5170402

Manne, V., Handa, P., and Kowdley, K. V. (2018). pathophysiology of nonalcoholic fatty liver disease/nonalcoholic steatohepatitis. Clin. Liver Dis. 22, 23-37. doi: 10.1016/j.cld.2017.08.007

Melo, C. A., Drost, J., Wijchers, P. J., van de Werken, H., de Wit, E., Vrielink, J. A. O., et al. (2013). eRNAs are required for p53-dependent enhancer activity and gene transcription. Mol. Cell 49, 524-535. doi: 10.1016/j.molcel.2012.11.021

Meng, H., and Bartholomew, B. (2017). Emerging roles of transcriptional enhancers in chromatin looping and promoter-proximal pausing Of RNA Polymerase II. J. Biol. Chem. 293, 13786-13794. doi: 10.1074/jbc.R117.813485
Miyaaki, H., Ichikawa, T., Kamo, Y., Taura, N., Honda, T., Shibata, H., et al. (2014). Significance of serum and hepatic microRNA-122 levels in patients with non-alcoholic fatty liver disease. Liver Int. 34, e302-e307. doi: 10.1111/liv.12429

O'Brien, J., Hayder, H., Zayed, Y., and Peng, C. (2018). Overview of MicroRNA biogenesis. Mechanisms of actions, and circulation. Front. Endocrinol. 9:402. doi: 10.3389/fendo.2018.00402

Ogawa, T., Enomoto, M., Fujii, H., Sekiya, Y., Yoshizato, K., Ikeda, K., et al. (2012). MicroRNA-221/222 upregulation indicates the activation of stellate cells and the progression of liver fibrosis. Gut 61, 1600-1609. doi: 10.1136/gutjnl-2011300717

Otsuka, M., Kishikawa, T., Yoshikawa, T., Yamagami, M., Ohno, M., Takata, A., et al. (2016). MicroRNAs and liver disease. J. Hum. Genet. 62:75. doi: 10.1038/ jhg.2016.53

Panzitt, K., Tschernatsch, M. M. O., Guelly, C., Moustafa, T., Stradner, M., Strohmaier, H. M., et al. (2007). Characterization of HULC, a novel gene with striking up-regulation in hepatocellular carcinoma, as noncoding RNA. Gastroenterology 132, 330-342. doi: 10.1053/j.gastro.2006.08.026

Paschos, G. K., Ibrahim, S., Song, W.-L., Kunieda, T., Grant, G., Reyes, T. M., et al. (2012). Obesity in mice with adipocyte-specific deletion of clock component Arntl. Nat. Med. 18, 1768-1777. doi: 10.1038/nm.2979

Peverill, W., Powell, L., and Skoien, R. (2014). Evolving concepts in the pathogenesis of NASH: beyond steatosis and inflammation. Int. J. Mol. Sci. 15:8591. doi: $10.3390 /$ ijms 15058591

Pirola, C. J., Gianotti, T. F., Castaño, G. O., Mallardi, P., Martino, J. S., Ledesma, M. M. G. L., et al. (2015). Circulating microRNA signature in non-alcoholic fatty liver disease: from serum non-coding RNAs to liver histology and disease pathogenesis. Gut 64, 800-812. doi: 10.1136/gutjnl-2014-306996

Qiu, L., Tang, Q., Li, G., and Chen, K. (2017). Long non-coding RNAs as biomarkers and therapeutic targets: recent insights into hepatocellular carcinoma. Life Sci. 191, 273-282. doi: 10.1016/j.lfs.2017.10.007

Quinn, J. J., and Chang, H. Y. (2015). Unique features of long non-coding RNA biogenesis and function. Nat. Rev. Genet. 17:47. doi: 10.1038/nrg.2015.10

Rayner, K. J., Suárez, Y., Dávalos, A., Parathath, S., Fitzgerald, M. L., Tamehiro, N., et al. (2010). MiR-33 contributes to the regulation of cholesterol homeostasis. Science 328, 1570-1573. doi: 10.1126/science.1189862

Rodgers, J. T., Lerin, C., Haas, W., Gygi, S. P., Spiegelman, B. M., and Puigserver, P. (2005). Nutrient control of glucose homeostasis through a complex of PGC- $1 \alpha$ and SIRT1. Nature 434:113. doi: 10.1038/nature03354

Roy, S., Benz, F., Luedde, T., and Roderburg, C. (2015). The role of miRNAs in the regulation of inflammatory processes during hepatofibrogenesis. Hepatobiliary Surg. Nutr. 4, 24-33. doi: 10.3978/j.issn.2304-3881.2015.01.05

Salvoza, N. C., Klinzing, D. C., Gopez-Cervantes, J., and Baclig, M. O. (2016). Association of circulating serum miR-34a and miR-122 with dyslipidemia among patients with non-alcoholic fatty liver disease. PLoS One 11:e0153497. doi: 10.1371/journal.pone.0153497

Sarkar, A., Volff, J.-N., and Vaury, C. (2017). piRNAs and their diverse roles: a transposable element-driven tactic for gene regulation? FASEB J. 31, 436-446. doi: 10.1096/fj.201600637RR

Shan, W., Gao, L., Zeng, W., Hu, Y., Wang, G., Li, M., et al. (2015). Activation of the SIRT1/p66shc antiapoptosis pathway via carnosic acid-induced inhibition of miR-34a protects rats against nonalcoholic fatty liver disease. Cell Death Amp 6:e1833. doi: 10.1038/cddis.2015.196

Shimba, S., Ogawa, T., Hitosugi, S., Ichihashi, Y., Nakadaira, Y., Kobayashi, M., et al. (2011). Deficient of a clock gene, Brain and Muscle Arnt-Like Protein-1 (BMAL1), induces dyslipidemia and ectopic fat formation. PLoS One 6:e25231. doi: 10.1371/journal.pone.0025231

Shiromoto, Y., Kuramochi-Miyagawa, S., Daiba, A., Chuma, S., Katanaya, A., Katsumata, A., et al. (2013). GPAT2, a mitochondrial outer membrane protein, in piRNA biogenesis in germline stem cells. RNA 19, 803-810. doi: 10.1261/rna. 038521.113

Streba, L. A. M., Vere, C. C., Rogoveanu, I., and Streba, C. T. (2015). Nonalcoholic fatty liver disease, metabolic risk factors, and hepatocellular carcinoma: an open question. World J. Gastroenterol. 21, 4103-4110. doi: 10.3748/wjg.v21.i14.4103

Su, Q., Kumar, V., Sud, N., and Mahato, R. I. (2018). Role of MicroRNAs in the pathogenesis and treatment of progressive liver injury in NAFLD and liver fibrosis. Adv. Drug Deliv. Rev. 129, 54-63. doi: 10.1016/j.addr.2018.01.009

Sumida, Y., Niki, E., Naito, Y., and Yoshikawa, T. (2013). Involvement of free radicals and oxidative stress in NAFLD/NASH. 
Free Radic. Res. 47, 869-880. doi: 10.3109/10715762.2013. 837577

Sun, C., Liu, X., Yi, Z., Xiao, X., Yang, M., Hu, G., et al. (2015). Genomewide analysis of long noncoding RNA expression profiles in patients with non-alcoholic fatty liver disease. IUBMB Life 67, 847-852. doi: 10.1002/iub.1442

Takahashi, K., Yan, I., Haga, H., and Patel, T. (2014). Long non-coding RNA in liver diseases. Hepatology 60, 744-753. doi: 10.1002/hep.27043

Tan, Y., Ge, G., Pan, T., Wen, D., and Gan, J. (2014). A pilot study of serum MicroRNAs panel as potential biomarkers for diagnosis of nonalcoholic fatty liver disease. PLoS One 9:e105192. doi: 10.1371/journal.pone.0105192

Tilg, H., and Moschen, A. R. (2010). Evolution of inflammation in nonalcoholic fatty liver disease: the multiple parallel hits hypothesis. Hepatology 52, 1836-1846. doi: 10.1002/hep.24001

Tryndyak, V. P., Latendresse, J. R., Montgomery, B., Ross, S. A., Beland, F. A., Rusyn, I., et al. (2012). Plasma microRNAs are sensitive indicators of interstrain differences in the severity of liver injury induced in mice by a cholineand folate-deficient diet. Toxicol. Appl. Pharmacol. 262, 52-59. doi: 10.1016/j. taap.2012.04.018

Turchinovich, A., Baranova, A., Drapkina, O., and Tonevitsky, A. (2018). Cell-free circulating nucleic acids as early biomarkers for NAFLD and NAFLD-associated disorders. Front. Physiol. 9:1256. doi: 10.3389/fphys.2018.01256

Vishnoi, A., and Rani, S. (2017). "MiRNA biogenesis and regulation of diseases: an overview," in MicroRNA Profiling: Methods and Protocols, ed. S. Rani (New York, NY: Springer), 1-10.

Wang, L., and Jordan, I. K. (2018). Transposable element activity, genome regulation and human health. Curr. Opin. Genet. Dev. 49, 25-33. doi: 10.1016/j. gde.2018.02.006

Wang, X., and Wang, J. (2018). High-content hydrogen water-induced downregulation of miR-136 alleviates non-alcoholic fatty liver disease by regulating Nrf2 via targeting MEG3. Biol. Chem. 399, 397-406. doi: 10.1515/ hsz-2017-0303

Ward, J., Kanchagar, C., Veksler-Lublinsky, I., Lee, R. C., McGill, M. R., Jaeschke, H., et al. (2014). Circulating microRNA profiles in human patients with acetaminophen hepatotoxicity or ischemic hepatitis. Proc. Natl. Acad. Sci. U.S.A. 111, 12169-12174. doi: 10.1073/pnas.1412608111

Ward, M., McEwan, C., Mills, J. D., and Janitz, M. (2015). Conservation and tissuespecific transcription patterns of long noncoding RNAs. J. Hum. Transcr. 1, 2-9. doi: 10.3109/23324015.2015.1077591

Weng, W., Li, H., and Goel, A. (2019). Piwi-interacting RNAs (piRNAs) and cancer: emerging biological concepts and potential clinical implications. Biochim. Biophys. Acta 1871, 160-169. doi: 10.1016/j.bbcan.2018.12.005

Wilusz Jeremy, E. (2018). A $360^{\circ}$ view of circular RNAs: from biogenesis to functions. Wiley Interdiscip. Rev. 9:e1478. doi: 10.1002/wrna.1478

Wong, C.-M., Tsang, F. H.-C., and Ng, I. O.-L. (2018). Non-coding RNAs in hepatocellular carcinoma: molecular functions and pathological implications. Nat. Rev. Gastroenterol. 15:137. doi: 10.1038/nrgastro.2017.169

Wruck, W., Graffmann, N., Kawala, M. A., and Adjaye, J. (2017). Concise review: current status and future directions on research related to nonalcoholic fatty liver disease. Stem Cells 35, 89-96. doi: 10.1002/stem.2454

Wu, J.-C., Luo, S.-Z., Liu, T., Lu, L.-G., and Xu, M.-Y. (2019). linc-SCRG1 accelerates liver fibrosis by decreasing RNA-binding protein tristetraprolin. FASEB J. 33, 2105-2115. doi: 10.1096/fj.201800098RR

Xu, Q., Li, Y., Shang, Y.-F., Wang, H.-L., and Yao, M.-X. (2015). miRNA-103: molecular link between insulin resistance and nonalcoholic fatty liver disease. World J. Gastroenterol. 21, 511-516. doi: 10.3748/wjg.v21.i2.511

Xu, Y., Zalzala, M., Xu, J., Li, Y., Yin, L., and Zhang, Y. (2015). A metabolic stressinducible miR-34a-HNF4 $\alpha$ pathway regulates lipid and lipoprotein metabolism. Nat. Commun. 6:7466. doi: 10.1038/ncomms8466

Yamada, H., Ohashi, K., Suzuki, K., Munetsuna, E., Ando, Y., Yamazaki, M., et al. (2015). Longitudinal study of circulating miR-122 in a rat model of nonalcoholic fatty liver disease. Clin. Chim. Acta 446, 267-271. doi: 10.1016/j.cca. 2015.05.002

Yamada, H., Suzuki, K., Ichino, N., Ando, Y., Sawada, A., Osakabe, K., et al. (2013). Associations between circulating microRNAs (miR-21, miR-34a, miR122 and miR-451) and non-alcoholic fatty liver. Clin. Chim. Acta 424, 99-103. doi: 10.1016/j.cca.2013.05.021

Yamamura, S., Imai-Sumida, M., Tanaka, Y., and Dahiya, R. (2018). Interaction and cross-talk between non-coding RNAs. Cell. Mol. Life Sci. 75, 467-484. doi: 10.1007/s00018-017-2626-6
Yao, T., Chen, Q., Fu, L., and Guo, J. (2017). Circular RNAs: biogenesis, properties, roles, and their relationships with liver diseases. Hepatol. Res. 47, 497-504. doi: 10.1111/hepr.12871

Younossi, Z., Anstee, Q. M., Marietti, M., Hardy, T., Henry, L., Eslam, M., et al. (2017). Global burden of NAFLD and NASH: trends, predictions, risk factors and prevention. Nat. Rev. Gastroenterol. 15:11. doi: 10.1038/nrgastro.2017.109

Younossi, Z. M., Koenig, A. B., Abdelatif, D., Fazel, Y., Henry, L., and Wymer, M. (2016). Global epidemiology of nonalcoholic fatty liver disease-meta-analytic assessment of prevalence, incidence, and outcomes. Hepatology 64, 73-84. doi: 10.1002/hep.28431

Yu, F., Chen, B., Dong, P., and Zheng, J. (2017a). HOTAIR epigenetically modulates PTEN expression via MicroRNA-29b: a novel mechanism in regulation of liver fibrosis. Mol. Ther. 25, 205-217. doi: 10.1016/j.ymthe.2016. 10.015

Yu, F., Jiang, Z., Chen, B., Dong, P., and Zheng, J. (2017b). NEAT1 accelerates the progression of liver fibrosis via regulation of microRNA-122 and Kruppel-like factor 6. J. Mol. Med. 95, 1191-1202. doi: 10.1007/s00109-017-1586-5

Yu, F., Geng, W., Dong, P., Huang, Z., and Zheng, J. (2018). LncRNA-MEG3 inhibits activation of hepatic stellate cells through SMO protein and miR-212. Cell Death Dis. 9:1014. doi: 10.1038/s41419-018-1068-x

Yu, F., Lu, Z., Cai, J., Huang, K., Chen, B., Li, G., et al. (2015a). MALAT1 functions as a competing endogenous RNA to mediate Racl expression by sequestering miR-101b in liver fibrosis. Cell Cycle 14, 3885-3896. doi: 10.1080/15384101. 2015.1120917

Yu, F., Zheng, J., Mao, Y., Dong, P., Li, G., Lu, Z., et al. (2015b). Long noncoding RNA APTR promotes the activation of hepatic stellate cells and the progression of liver fibrosis. Biochem. Biophys. Res. Commun. 463, 679-685. doi: 10.1016/j.bbrc.2015.05.124

Yu, F., Lu, Z., Chen, B., Dong, P., and Zheng, J. (2016). Identification of a novel lincRNA-p21-miR-181b-PTEN signaling cascade in liver fibrosis. Mediators Inflamm. 2016:9856538. doi: 10.1155/2016/9856538

Zhang, K., Han, X., Zhang, Z., Zheng, L., Hu, Z., Yao, Q., et al. (2017). The liverenriched lnc-LFAR1 promotes liver fibrosis by activating TGF $\beta$ and Notch pathways. Nat. Commun. 8:144. doi: 10.1038/s41467-017-00204-4

Zhang, M., Chi, X., Qu, N., and Wang, C. (2018). Long noncoding RNA IncARSR promotes hepatic lipogenesis via Akt/SREBP-1c pathway and contributes to the pathogenesis of nonalcoholic steatohepatitis. Biochem. Biophys. Res. Commun. 499, 66-70. doi: 10.1016/j.bbrc.2018.03.127

Zheng, J., Dong, P., Mao, Y., Chen, S., Wu, X., Li, G., et al. (2015). lincRNA-p21 inhibits hepatic stellate cell activation and liver fibrogenesis via p21. FEBS J. 282, 4810-4821. doi: 10.1111/febs.13544

Zheng, J., Yu, F., Dong, P., Wu, L., Zhang, Y., Hu, Y., et al. (2016). Long noncoding RNA PVT1 activates hepatic stellate cells through competitively binding microRNA-152. Oncotarget 7, 62886-62897. doi: 10.18632/oncotarget.11709

Zhou, B., Yuan, W., and Li, X. (2018). LncRNA Gm5091 alleviates alcoholic hepatic fibrosis by sponging miR-27b/23b/24 in mice. Cell Biol. Int. 42, 1330-1339. doi: 10.1002/cbin.11021

Zhou, Y., Lv, X., Qu, H., Zhao, K., Fu, L., Zhu, L., et al. (2018). Preliminary screening and functional analysis of circular RNAs associated with hepatic stellate cell activation. Gene 677, 317-323. doi: 10.1016/j.gene.2018.08.052

Zhu, J., Luo, Z., Pan, Y., Zheng, W., Li, W., Zhang, Z., et al. (2018). H19/miR$148 \mathrm{a} /$ USP4 axis facilitates liver fibrosis by enhancing TGF- $\beta$ signaling in both hepatic stellate cells and hepatocytes. J. Cell. Physiol. 234, 9698-9710. doi: $10.1002 /$ jcp. 27656

Zhu, L., Ren, T., Zhu, Z., Cheng, M., Mou, Q., Mu, M., et al. (2018). Thymosin$\beta 4$ mediates hepatic stellate cell activation by interfering with CircRNA0067835/miR-155/FoxO3 signaling pathway. Cell. Physiol. Biochem. 51, 1389-1398. doi: 10.1159/000495556

Conflict of Interest Statement: The authors declare that the research was conducted in the absence of any commercial or financial relationships that could be construed as a potential conflict of interest.

Copyright (c) 2019 Sulaiman, Muhsin and Jamal. This is an open-access article distributed under the terms of the Creative Commons Attribution License (CC BY). The use, distribution or reproduction in other forums is permitted, provided the original author(s) and the copyright owner(s) are credited and that the original publication in this journal is cited, in accordance with accepted academic practice. No use, distribution or reproduction is permitted which does not comply with these terms. 